\title{
The incidence of injuries and exposure time of professional football club players in the Premier Soccer League during football season
}

\author{
T Calligeris, ${ }^{1,3}$ BSc Hons (Physio), MPhil (Sports Physio); T Burgess, ${ }^{2,3}$ BSc (Physio), PhD; M Lambert ${ }^{2}$ PhD \\ ${ }^{1}$ Sports Science Physiotherapy Centre, Sports Science Institute of South Africa, Cape Town, South Africa \\ ${ }^{2}$ Division of Exercise Science and Sports Medicine, Department of Human Biology, Faculty of Health Sciences, University of Cape Town, South Africa \\ ${ }^{3}$ Division of Physiotherapy, Department of Health and Rehabilitation Sciences, University of Cape Town, South Africa
}

Corresponding author: T Calligeris (theo@sspc.co.za)

\begin{abstract}
Background. Data on the incidence of football injuries and exposure time of players during matches and training in the South African (SA) Premier Soccer League (PSL) are lacking.

Objective. To calculate the incidence of injuries and the exposure time (practice and match) of the players of a PSL team over a full season. Methods. Injury and training data of the players in the squad $(N=32)$ were recorded on a daily basis by the medical support staff, according to the methods of the Football Medical Assessment and Research Centre (F-MARC) protocol.

Results. One hundred and thirty injuries were recorded in the season. The most affected body parts were the thigh $(n=27,21 \%)$ and ankle $(n=27,21 \%)$. The overall incidence was 13.4 injuries per 1000 player-hours. The incidence during training was 6.6 injuries/1 000 playerhours and during matches 88.9 injuries/1 000 player-hours. The most frequent injury was haematoma/contusion/bruising $(n=43,33 \%)$. Of the total injuries, $12 \%$ were recurrent. Injury through contact with another player was high (62\%). Seventy-six per cent of the injuries were not associated with any violation of the laws of the game. The average time off due to injury was 8 days. The total exposure time over the full season resulted in a combined average of 18162 minutes ( 303 hours).

Conclusion. These data differ from the data in European studies. Injury and exposure data measured throughout the season have the potential to identify risks and mechanisms of injuries. This study highlights the necessity for all clubs in the PSL to adopt a standardised injury monitoring programme, using standardised methodology, so that the management of professional players in SA may be improved.
\end{abstract}

S Afr J Sports Med 2015;27(1):16-19. DOI:10.7196/SAJSM.610

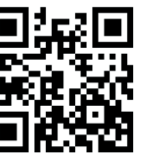

Football, also known as soccer, is the most widely played sport in the world, with 240 million people playing regularly in more than 200 countries. ${ }^{[1]}$ The science of football started emerging in the 1970s; however, there were not many international research papers published during this era. By the 1990s, there were further research developments and more papers published. A review of 20 epidemiological studies on adult soccer players showed that epidemiological information with regard to soccer injuries was inconsistent and incomplete. ${ }^{[1]}$ Since the 1990s, more papers have been published, showing how the science of football and medical support structures of teams have evolved..$^{[2,3]}$ This proliferation of knowledge has led to the establishment of the independent research body of the Fédération Internationale de Football Association (FIFA) called F-MARC (Football Medical Assessment and Research Centre) in 1994, uniting an international group of experts in football medicine.

In South Africa (SA) the application of scientific knowledge on the description and prevention of injuries in football is lacking at all levels. There are poorly developed support structures, particularly at school and university level, and the growth of applied science of the local game has been lacking. For example, a PubMed search using the term 'soccer football South Africa' revealed four results, 'soccer South Africa' revealed 36 results and 'football South Africa' displayed 54 results, of which the majority were related to rugby football. The articles on football made no mention of the Premier Soccer League (PSL) in SA. This is unfortunate, because research on football in SA is a necessary tool required to answer questions that are specific to the demands of the game in SA. Without answering these questions, the rate of improvement in the local game will be curtailed. Also, without data on injuries in the PSL, it is difficult to determine whether any changes occur after intervention in any aspect of a player's management.

Although many studies have been conducted on players in Europe, these data may not necessarily apply to players in SA. Therefore, the objective of this study was to describe the incidence of injuries and exposure time of players in a professional football club in the PSL over a full season, using a standardised method of collecting data. ${ }^{[4]}$ An understanding of injury characteristics in SA football may contribute towards developing injury-prevention strategies and possible rule changes that may also reduce the risk of injury.

\section{Methods}

The study had a retrospective cohort design. Participants gave their informed consent for their data to be analysed for the study after the nature, purpose and procedures were thoroughly explained. The study was approved by the Human Research Ethics Committee of the University of Cape Town (HREC REF 308/2010). Participants included healthy, active male professional footballers who played for a PSL 1st team and reserve team (Ajax). A player baseline information form was completed by the chief club physiotherapist in consultation with the players. Whenever a player in an official club match or practice was 
injured, the details and circumstances about the injury were recorded immediately by the chief club physiotherapist, according to the F-MARC injury report form. ${ }^{[4]}$ An injury was defined as any 'physical complaint sustained by a player that resulted from a football match or football training, irrespective of the need for medical attention or time loss from football activities. An injury that results in a player receiving medical attention is referred to as a 'medical attention' injury, and an injury that results in a player being unable to take a full part in future football training or match play as a 'time loss' injury. ${ }^{[4]}$

The exposure time was calculated from the total number of hours of participation per day, defined according to the following categories:

- Normal field training session with the coach and manager

- Recovery session in the gym comprising spinning, hydro-exercises and stretching

- Conditioning session

- Endurance session

- High-performance testing session.

All injuries during the season were expressed as injuries/1 000 player-hours, as described by Brooks and Fuller. ${ }^{[5]}$

The data are presented as absolute numbers, percentages, averages ( $95 \%$ confidence intervals (CIs)) or means (standard deviations $(\mathrm{SDs}))$, as appropriate.

\section{Results}

\section{Description of players}

A total of 32 players participated in the study. The average (SD) age of the players was 24 (2) years. Average height and weight were $1.8(0.1) \mathrm{m}$ and $75.6(4.1) \mathrm{kg}$, respectively.

\section{Quantification of exposure type}

The players spent on average $67 \%$ of their time training, $10 \%$ playing matches, $13 \%$ in recovery training and $9 \%$ in conditioning training (Table 1). The remainder of the time was spent doing endurance training or testing. There were 236 training sessions over the season.

\section{Injuries}

Over the whole season, 130 injuries were documented, comprising 15 preseason injuries (12\%) and 115 inseason injuries (Fig. 1). Four injuries did not have a 'time off' value and were excluded from the data on time off from training and competition.

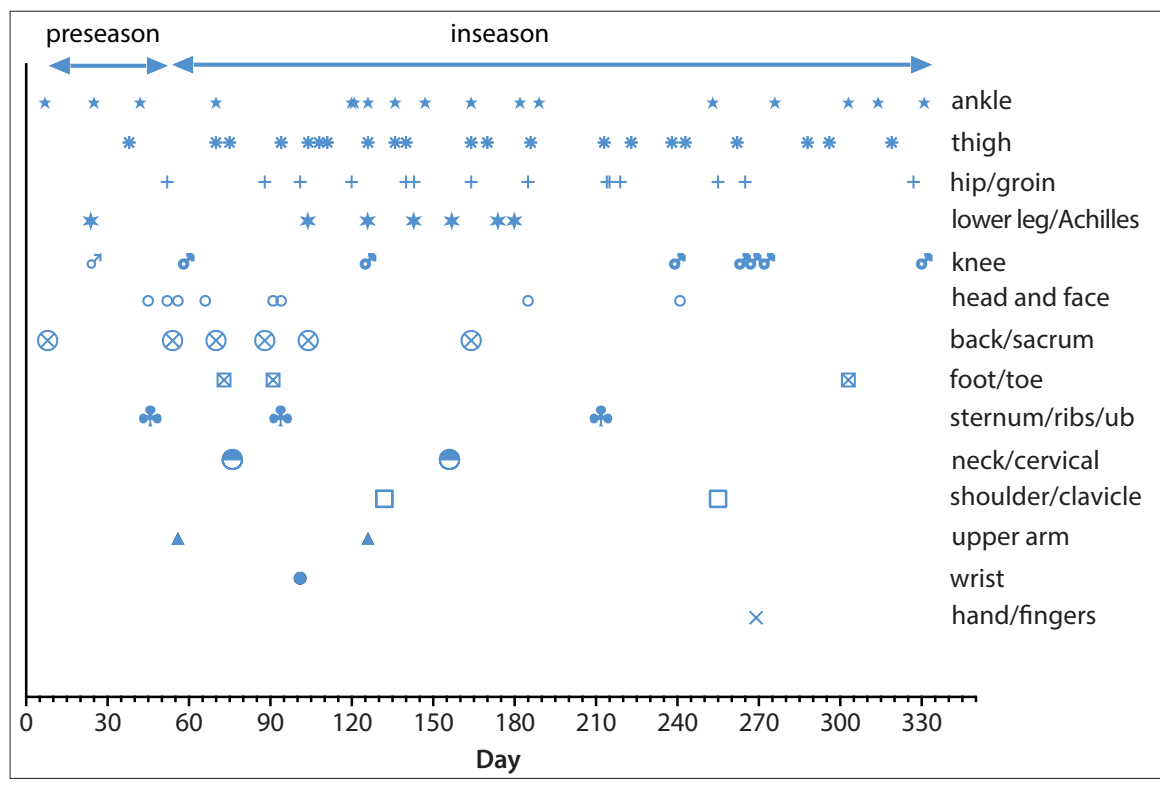

Fig. 1. Distribution of the 130 injuries that occurred during preseason ( 0 - 47 days) and inseason (48 - 341 days), showing the body parts that were injured. The body parts are on the right of the figure with the symbol to the left, e.g. hand/fingers is represented by the symbol $\times$. (ub $=$ upper back.)

Table 1. Total different exposure times per player over one season

\begin{tabular}{|c|c|c|c|c|c|c|c|}
\hline & \multicolumn{7}{|c|}{ Total time (min) } \\
\hline & Training & Match & Recovery & Conditioning & Endurance & Testing & Total \\
\hline Mean & 12252 & 1834 & 2324 & 1701 & 39 & 12 & $18162^{*}$ \\
\hline$-95 \%$ & 10610 & 1457 & 2006 & 1454 & 24 & 9 & 15748 \\
\hline $95 \%$ & 13894 & 2211 & 2642 & 1949 & 53 & 15 & 20575 \\
\hline
\end{tabular}

The overall injury rate was 13.4/1 000 playerhours (95\% CI 11.1 - 15.7). Lower rates were found in training (6.6/1 000 player-hours; $95 \%$ CI 4.6 - 8.6) as opposed to matches (88.9/1 000 player-hours; 95\% CI 70.2 107.6). The nature of the injuries varied; however, the most common body parts injured were the thighs $(n=27)$ and the ankles ( $n=27)$ (Table 2).

The average time off for all injuries was 8 days. Minor injuries ( $<7$ days) accounted for $18 \%$, moderate injuries ( 8 - 28 days) $26 \%$ and severe injuries (>28 days) $56 \%$ of the injuries. Two per cent of the days during the season were lost because of minor injuries, $7 \%$ were lost owing to moderate injuries and $23 \%$ were lost to severe injuries.

Sixty-five injuries occurred on the nondominant side and 57 injuries on the dominant side of the body. Eight injuries were sustained on other parts of the body (head and spine). The average time off following an injury to the non-dominant side was 10 days
Table 2. Injuries according to body part ${ }^{\star}$

\begin{tabular}{ll}
\hline Body part & $\boldsymbol{n}(\%)$ \\
\hline Thigh & $27(21)$ \\
Ankle & $27(21)$ \\
Hip/groin & $16(12)$ \\
Lower leg/Achilles tendon & $15(12)$ \\
Knee & $11(8)$ \\
Head/face & $10(8)$ \\
Low back/sacrum/pelvis & $8(6)$ \\
Foot/toe & $5(4)$ \\
Sternum/ribs/upper back & $3(2)$ \\
Neck/cervical spine & $2(2)$ \\
Shoulder/clavicle & $2(2)$ \\
Upper arm & $2(2)$ \\
Wrist & $1(1)$ \\
Hand/finger/thumb & $1(1)$ \\
All groups & $130(100)$ \\
$*$ Dota &
\end{tabular}

${ }^{*}$ Data are expressed as a per cent of the total number of injuries. 
Table 3. Type of injuries sustained ${ }^{\star}$

\begin{tabular}{ll}
\hline & $\boldsymbol{n}(\%)$ \\
\hline Haematoma/contusion/bruise & $43(33)$ \\
Muscle rupture/strain/tear/cramp & $38(29)$ \\
Sprain/ligament injury & $30(23)$ \\
Other bone injury & $7(5)$ \\
Tendon injury/rupture/tendinosis/bursitis & $2(2)$ \\
Concussion with/without loss of consciousness & $2(2)$ \\
Abrasion & $2(2)$ \\
Lesion meniscus/cartilage & $2(2)$ \\
Laceration & $2(2)$ \\
Fracture & $1(1)$ \\
Other & $1(1)$ \\
Total injuries & $130(100)$ \\
${ }^{*}$ Data are expressed as a per cent of the total number of injuries. &
\end{tabular}

Table 4. Injuries sustained by violation of the laws ${ }^{\star}$

\begin{tabular}{ll}
\hline & $\boldsymbol{n}(\%)$ \\
\hline No violation of the laws & $99(76)$ \\
Violation resulting in free kick/penalty & $24(18)$ \\
Violation resulting in yellow card & $6(5)$ \\
Violation resulting in red card & $1(1)$ \\
Total injuries & $130(100)$ \\
${ }^{*}$ Data are expressed as a per cent of the total number of injuries. &
\end{tabular}

(95\% CI 6 - 14) and to the dominant side 6 days (95\% CI 4 - 8). The majority of the players were right-foot dominant.

The longest average time off occurred with sternum/ribs/upper back injuries ( 23 days, range $5-38 ; n=3$ ), followed by thigh injuries (11 days, range 2 - 79; $n=27)$, hip/groin injuries (11 days, range $1-41 ; n=14)$ and ankle injuries (10 days, range $2-57 ; n=27)$. The most common type of injury was a haematoma/contusion/bruise, representing $33 \%$ of all injuries (Table 3 ).

Midfielders were most affected by haematoma/contusion/bruisetype injuries $(n=22)$, with a mean time off from training and competition of 4 (5) days. They were also more likely to sustain an injury, recurrent or non-recurrent, than any other player position, while goalkeepers sustained the least number of recurrent and nonrecurrent injuries. More injuries were first-time injuries ( $n=114,88 \%)$, with the remainder being concurrent $(n=16,12 \%)$. Four players did not return to play owing to the severity of the injury or were transferred to another club; all four sustained non-recurrent injuries.

Seventy-seven per cent $(n=99)$ of the injuries were a result of trauma, while $23 \%(n=30)$ were related to overuse. Defenders sustained more overuse injuries $(n=13,30 \%)$ than midfielders $(n=11,19 \%)$. Forwards were more likely to sustain an injury owing to trauma $(n=16,76 \%)$ than to overuse $(n=5,24 \%)$. More injuries occurred during matches $(n=87$, $67 \%)$ than in training $(n=43 ; 33 \%)$ over the full PSL season. Of the total injuries, midfielders sustained most of the match injuries, followed by defenders, forwards and goalkeepers. The same pattern was seen in training injuries. A total of 43 (33\%) non-contact and 87 (67\%) contact injuries were recorded over the full season. Of all the injuries over the season, $24 \%(n=31)$ were as a result of a violation of the laws (Table 4).

\section{Discussion}

The first finding of this study was that the mean training and match exposure times per player during the season were 12252 minutes ( 204 hours) and 1834 minutes ( 31 hours), respectively. This can be compared with European studies, which have shown that the mean training and match exposure times per player are 15720 minutes (262 hours) and 2400 minutes (40 hours), respectively. ${ }^{[6]}$ This suggests that professional European players are exposed to more football per annum than SA players, which has the potential to affect injuries and cumulative improvement in the level of performance during a season.

The second finding of this study was that there were 6.6 injuries/ 1000 player-hours during training and 88.9 injuries/1 000 playerhours during matches. Twelve per cent of all injuries were sustained in the preseason phase of training. Studies in Europe have shown that preseason injuries contribute to $17 \%$ of the total number of injuries. ${ }^{[7]}$

It may be argued that preseason injuries are fewer in SA owing to the lower exposure times, but a counterargument to this is that SA players are less prepared for the inseason phase and may therefore be at a greater risk of inseason injuries owing to poor preparation. Further research needs to be done to clarify the interpretation.

The fact that most injuries occurred at the beginning and end of the season may be owing to lack of match fitness, strength and conditioning early in the season, and the cumulative effect of repeated and prolonged exposure to football at the end of the season. The latter may have been exacerbated by the demand from the PSL to complete the league schedule sooner than usual to accommodate the FIFA World Cup in 2010.

The average time off in this study was 8 days, compared with other studies, which describe 17 days ${ }^{[8]}$ and 18 days ${ }^{[9]}$ This can be interpreted in one of two ways: either the injuries of the European players were more serious, or the medical care and management approach of the club towards the player was different. Once again, further research will need to identify the correct interpretation. This has important clinical implications that affect the management of the players. Certain body parts have been shown to have a high risk of injury with a high time loss factor, e.g. thigh $(n=27)$, with a maximum time off of 79 days. These findings are consistent with other studies. ${ }^{[10]}$

Non-dominant body side injuries ( $n=65)$ occurred slightly more frequently than injuries on the dominant side $(n=57)$. Other studies have shown contrasting results. ${ }^{[1]}$ In this study, an interesting observation showed that players took a longer time to return to play if an injury was sustained to the non-dominant side of the body as opposed to the dominant side. Once again, this has important clinical implications for management.

A study of European football showed a significantly higher incidence of overuse injuries in the preseason compared with injuries arising from trauma ( $68 \%$ v. $29 \%$, respectively). The percentage of contact injuries during preseason was significantly less than inseason ( $29 \%$ v. $40 \%$, respectively). ${ }^{[7]}$ The percentage of overuse injuries sustained inseason was also greater than traumatic injuries (56\% v. $40 \%$, respectively). ${ }^{[7]}$ However, different mechanisms of injury were observed in this study. The possible reasons for the different types 
of injury are multidimensional. For example, factors responsible for this injury pattern may include the different style of play (African v. European), vision of the game, skill, difference in fitness levels, player's strength, difference in preparation and training methods, nutrition and the mental state of players. Further studies are required to determine specific contributing factors in SA football.

Recurrent injuries are part of the season injury profile, although they represent a smaller part of the profile (12\%). The relatively small percentage of recurrent injuries may be related to better management of injured players owing to epidemiological studies that have described the risks of injuries ${ }^{[12]}$ and of sustaining recurrent injuries. ${ }^{[6]}$ For example, more focus is placed on preventing injuries through correct warm-up $^{[13]}$ and exercise programmes. ${ }^{[14]}$ It has been shown that adopting these principles results in a reduction of recurrent injuries. The midfielders and defenders in this study sustained the most recurrent injuries. Identifying the injuries and risks, and intervening with prevention programmes, may reduce the risk of injury in the following season..$^{[13,15]}$ However, most injury prevention programmes may assist in reducing the risk of overuse injuries. Trauma is due to direct contact. Therefore increasing awareness of these type of injuries, strengthening high-risk contact areas on the body, improving proprioception, agility, coordination, neuromuscular control, ${ }^{[16]}$ reaction-response time (reflex-reaction exercises) ${ }^{[17]}$ and core stability ${ }^{[18]}$ may reduce the incidence of contact injuries.

The injury patterns differed depending on the players' position. In this study, midfielders sustained the most injuries compared with any other player position. A similar injury pattern was described in female professional players participating in the Women's United Soccer Association league. ${ }^{[19]}$ However, another study on African players showed that defenders sustained the most injuries over a full season, followed by forwards, midfielders and goalkeepers. ${ }^{[20]}$

In this study, $24 \%$ of all injuries sustained over a season were owing to unfair play. Inconsistent refereeing during the season may compound this. ${ }^{[21]}$ These statistics may support the call for a video referee, and education for both players and referees. There may also be a need to improve the laws of the game to protect players. ${ }^{[22]}$ No studies have conclusively described the injuries sustained by violation of the laws. This is an important part of assessing the manner in which the injury is sustained and requires further research.

\section{Conclusion}

The importance of assessing the incidence of injury and exposure times of footballers playing in a club cannot be underestimated. A better understanding of the underlying risk factors, exposure to injury and mechanisms of injury in SA football players should be promoted. It is therefore strongly suggested that a standardised injury surveillance protocol be established in all the teams in the PSL; this protocol should run through the different leagues under the jurisdiction of the SA Football Association (SAFA). User-friendly software programs have been designed specifically for this purpose; therefore, injury surveillance using these protocols should become standard practice for all teams.

Acknowledgements. The authors would like to acknowledge Mr George Comitis (CEO of Ajax Cape Town (CT) football club at the time) who supported the study, Mr Gavin Benjefield, Mr Lorenzo Meyer, Mr Craig von Weilligh (Ajax CT Medical Team), who assisted with data collection, and the players of Ajax CT who participated in the study.

\section{References}

1. Maffulli N, Caine DJ, eds. Epidemiology of Pediatric Sports Injuries: Team Sports. Basel, Switzerland: Karger, 2005

2. Ekstrand J, Tropp H. The incidence of ankle sprains in soccer. Foot Ankle 1990;11(1):41-44. [http://dx.doi.org/10.1177/107110079001100108]

3. Ekstrand J, Waldén M, Hägglund M. A congested football calendar and the wellbeing of players: Correlation between match exposure of European footballers before the World Cup 2002 and their injuries and performances during that World Cup. Br J Sports Med 2004;38(4):493-497. [http://dx.doi.org/10.1136/bjsm.2003.009134]

4. Fuller CW, Ekstrand J, Junge A, et al. Consensus statement on injury definitions and data collection procedures in studies of football (soccer) injuries. Br J Sports Med 2006;40(3):193-201. [http://dx.doi.org/10.1136/bjsm.2005.025270]

5. Brooks JH, Fuller CW. The influence of methodological issues on the results and conclusions from epidemiological studies of sports injuries: Illustrative examples. Sports Med 2006;36(6):459-472. [http://dx.doi.org/10.2165/00007256-20063606000001]

6. Hägglund M, Waldén M, Ekstrand J. Previous injury as a risk factor for injury in elite football: A prospective study over two consecutive seasons. Br J Sports Med 2006;40(9):767-772. [http://dx.doi.org/10.1136/bjsm.2006.026609]

7. Woods C, Hawkins R, Hulse M, Hodson A. The Football Association Medical Research Programme: An audit of injuries in professional football-analysis of preseason injuries. Br J Sports Med 2002;36(6):436-441. [http://dx.doi.org/10.1136/ bjsm.36.6.436]

8. Cromwell F, Walsh J, Gormle J. A pilot study examining injuries in elite Gaelic footballers. Br J Sports Med 2000;34(2):104-108. [http://dx.doi.org/10.1136/ bjsm.34.2.104]

9. Ekstrand J, Hägglund M, Waldén M. Injury incidence and injury patterns in professional football: The UEFA injury study. Br J Sports Med 2011;45(7):553-558. [http://dx.doi.org/10.1136/bjsm.2009.060582]

10. Dvorak J, Junge A, Derman W, Schwellnus M. Injuries and illnesses of football players during the 2010 FIFA World Cup. Br J Sports Med 2011;45(8):626-630. [http://dx.doi. org/10.1136/bjsm.2010.079905]

11. Hawkins RD, Fuller CW. A prospective epidemiological study of injuries in four English professional football clubs. Br J Sports Med 1999;33(3):196-203. [http:// dx.doi.org/10.1136/bjsm.33.3.196]

12. Arnason A, Sigurdsson SB, Gudmundsson A, Holme I, Engebretsen L, Bahr R. Risk factors for injuries in football. Am J Sports Med 2004;32(Suppl 1):S5-S16. [http:// dx.doi.org/10.1177/0363546503258912]

13. Soligard T, Myklebust G, Steffen K, et al. Comprehensive warm-up programme to prevent injuries in young female footballers: Cluster randomised controlled trial. BMJ 2008;337:a2469. [http://dx.doi.org/10.1136/bmj.a2469]

14. Dvorak J, Junge A, Chomiak J, et al. Risk factor analysis for injuries in football players. Possibilities for a prevention program. Am J Sports Med 2000;28(5):69-74. [http:// dx.doi.org/10.1177/28.suppl_5.S-69]

15. Soligard T, Nilstad A, Steffen K, et al. Compliance with a comprehensive warm-up programme to prevent injuries in youth football. Br J Sports Med 2010;44(11):787793. [http://dx.doi.org/10.1136/bjsm.2009.070672]

16. Filipa A, Byrnes R, Paterno MV, Myer GD, Hewett TE. Neuromuscular training improves performance on the star excursion balance test in young female athletes. J Orthop Sports Phys Ther 2010;40(9):551-558. [http://dx.doi.org/10.2519/jospt.2010.3325]

17. Borghuis AJ, Lemmink KA, Hof AL. Core muscle response times and postural reactions in soccer players and nonplayers. Med Sci Sports Exerc 2011;43(1):108114. [http://dx.doi.org/10.1249/MSS.0b013e3181e93492]

18. Leetun DT, Ireland ML, Willson JD, Ballantyne BT, Davis IM. Core stability measures as risk factors for lower extremity injury in athletes. Med Science Sports Exerc 2004;36(6):926-934. [http://dx.doi.org/10.1249/01.MSS.0000128145.75199.C3]

19. Giza E, Mithofer K, Farrell L, Zarins B, Gill T. Injuries in women's professional soccer. Br J Sports Med 2005;39(4):212-216. [http://dx.doi.org/10.1136/ bjsm.2004.011973]

20. Azubuike SO, Okojie OH. An epidemiological study of football (soccer) injuries in Benin City, Nigeria. Br J Sports Med 2009;43(5):382-386. [http://dx.doi.org/10.1136/ bjsm.2008.051565]

21. Fuller CW Smith GL, Junge A, Dvorak J. An assessment of player error as an injury causation factor in international football. Am J Sports Med 2004;32(1):S28-S35. [http://dx.doi.org/10.1177/0363546503261247]

22. Andersen TE, Engebretsen L, Bahr R. Rule violations as a cause of injuries in male Norwegian professional football: Are the referees doing their job? Am J Sports Med 2004;32(1):S62-S8. [http://dx.doi.org/10.1177/0363546503261412] 